Forum 2019 · 34:193

https://doi.org/10.1007/s12312-019-0562-0

Online publiziert: 14. Februar 2019

C) Springer Medizin Verlag $\mathrm{GmbH}$, ein Teil von Springer Nature 2019

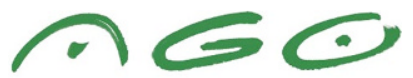

ARBEITSGEMEINSCHAFT

GYNÄKOLOGISCHE

ONKOLOGIE E. V.

Arbeitsgemeinschaft Gynäkologische Onkologie (AGO)

\title{
Neuer Vorsitzender der AGO J.-U. Blohmer stellt sich vor
}

für Gynäkologische Onkologie e. V.) und der Berliner Krebsgesellschaft und Vorsitzender des Fördervereins der Berliner Brustzentren. Darüber hinaus bin ich Mitglied des erweiterten Vorstandes der AWO-Gyn (Arbeitsgemeinschaft für ästhetische, plastische und wiederherstellende Operationsverfahren in der Gynäkologie e. V.) und DEGUM III (Deutsche Gesellschaft für Ultraschall in der Medizin e. V.), Mitglied des Arbeitskreises Mammasonographie.

\section{Die Ziele des Vorstandes}

Die Ziele des Vorstandes (Prof. Blohmer, Frau Prof. Hasenburg, Prof. Janni) sind insbesondere die Förderung und Gewinnung des klinischen und wissenschaftlichen Nachwuchses auf dem Gebiet der Gynäkologischen Onkologie, die internationale Zusammenarbeit und die Annahme und Umsetzung der Herausforderungen der Digitalisierung.

Die sehr erfolgreichen Projekte der bisherigen AGO-Vorstände werden selbstverständlich fortgeführt werden. Alle Organkommissionen der AGO (Mamma, Ovar, Uterus, Vagina/Vulva, Trafo, IMED) werden unterstützt und deren Tätigkeit koordiniert. Die etablierten Veranstaltungen wie die Jahrestagung der AGO und die Vorstellungen der neuen Leitlinien werden ebenfalls fortgeführt. An der Erweiterung der Apps zu den Therapie-

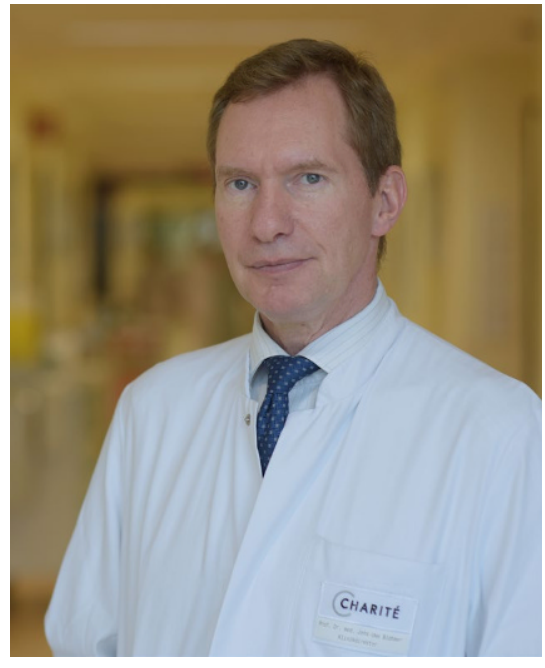

$\Delta$ Prof. Dr. med. Jens-Uwe Blohmer (৫ Wiebke Peitz, Charité)

Empfehlungen der AGO-Kommissionen wird gearbeitet werden, ebenso wie an der Weiterentwicklung und Aktualisierungen bestehender S3-Leitlinien.

Prof. Dr. med. J.-U. Blohmer, Klinikleiter

\section{Korrespondenzadresse}

Prof. Dr. med. J.-U. Blohmer

Gynäkologie mit Brustzentrum,

Charité - Universitätsmedizin Berlin

Charitéplatz 1, 10117 Berlin, Deutschland

jens.blohmer@charite.de 